PROCEEDINGS OF THE

AMERICAN MATHEMATICAL SOCIETY

Volume 125, Number 8, August 1997, Pages 2347-2352

S 0002-9939(97)04010-0

\title{
ON THE GENERALIZED STEPANOV THEOREM
}

\author{
MACIEJ KOCAN AND XU-JIA WANG
}

(Communicated by J. Marshall Ash)

\begin{abstract}
The generalized Stepanov theorem is derived from the Alexandrov theorem on the twice differentiability of convex functions. A parabolic version of the generalized Stepanov theorem is also proved.
\end{abstract}

In the first part of this note we provide a new proof of the generalized Stepanov theorem. This classical result is due to Calderón and Zygmund [3] (see also Oliver [12]), but is usually associated with Stepanov's name because it generalizes the Stepanov theorem (see e.g. [6]). The result we prove below (Theorem 1) constitutes a special case of a general theorem in [3]. Recently this particular version found applications in proving the twice differentiability a.e. of viscosity solutions of elliptic partial differential equations, see [11], [14] and [2]. The only complete proof of the generalized Stepanov theorem the authors are aware of is contained in [3], where Whitney's extension theorem is used. In this note the generalized Stepanov theorem will be proved by means of the Aleksandrov theorem on the twice differentiability of convex functions [1]; see also [5], [9], [10] or the appendix in [4] for more modern treatments.

In the second part of this note we show how to modify our proof to obtain a parabolic version of the generalized Stepanov theorem (Theorem 3). A result of this type is needed to prove the differentiability a.e. twice in $x$ and once in $t$ of viscosity solutions of parabolic equations. To the best of the authors' knowledge this result is original, though some relevant arguments appear in [16].

$|\cdot|$ and $\langle\cdot, \cdot\rangle$ will stand for the Euclidean norm and inner-product in $\mathbb{R}^{n}$, and $B_{r}(x)$ will denote the open ball in $\mathbb{R}^{n}$ of radius $r$ centered at $x$. Given a measurable set $A$ in an Euclidean space, $|A|$ will denote its Lebesgue measure.

Recall some notation from [3] (see also [17]). Let $u: \Omega \rightarrow \mathbb{R}, \Omega \subset \mathbb{R}^{n}$, be bounded and $x \in \Omega$. We say that $u \in T_{\infty}^{2}(x)\left(u \in t_{\infty}^{2}(x)\right.$, resp.) if there exists an affine function $P_{x}$ (a quadratic function $Q_{x}$ ) such that

$$
\begin{gathered}
\sup _{y \in B_{r}(x) \cap \Omega}\left|u(y)-P_{x}(y)\right| \leq O\left(r^{2}\right) \\
\left(\sup _{y \in B_{r}(x) \cap \Omega}\left|u(y)-Q_{x}(y)\right| \leq o\left(r^{2}\right) \text { as } r \downarrow 0, \text { resp. }\right) .
\end{gathered}
$$

Observe that $u \in t_{\infty}^{2}(x)$ if and only if $u$ possesses a second order Taylor series expansion at $x$ whose remainder behaves like $o\left(r^{2}\right)$. If this is the case we will say

Received by the editors February 21, 1996.

1991 Mathematics Subject Classification. Primary 26 B05.

This work was supported by the Australian Research Council.

(C) 1997 American Mathematical Society 
that $u$ is twice differentiable at $x$. On the other hand, $u \in T_{\infty}^{2}(x)$ is equivalent to saying that $u$ can be enclosed between two paraboloids meeting at $x$. In particular, if $\Omega \subset \mathbb{R}^{n}$ is open and $u \in T_{\infty}^{2}(x)$, then $u$ is differentiable at $x$ and $P_{x}(y)=$ $u(x)+\langle D u(x), y-x\rangle$.

Theorem 1 (Calderón-Zygmund [3]). Let $\Omega \subset \mathbb{R}^{n}$ be open and bounded and suppose that $u: \bar{\Omega} \rightarrow \mathbb{R}$ is bounded. If $u \in T_{\infty}^{2}(x)$ for a.e. $x \in \Omega$ then $u \in t_{\infty}^{2}(x)$ for a.e. $x \in \Omega$.

Proof. By the assumption for a.e. $x \in \Omega$ there are $p_{x} \in \mathbb{R}^{n}$ and $M_{x} \geq 0$ such that

$$
\left|u(y)-u(x)-\left\langle p_{x}, y-x\right\rangle\right| \leq M_{x}|y-x|^{2} \text { for all } y \in \Omega
$$

note that $p_{x}$ is uniquely determined and we can assume that $M_{x}$ is the smallest with this property. It follows that $M_{x}$ is well defined and finite a.e., moreover, the mapping $x \mapsto M_{x}$ is measurable. For $M=1,2, \ldots$ put

$$
\Omega_{M}=\left\{x \in \Omega: M_{x} \leq M\right\}
$$

then every $\Omega_{M}$ is measurable and $\cup_{M=1}^{\infty} \Omega_{M}$ is of full measure in $\Omega$. Therefore it is enough to show that for every $M$

$$
u \in t_{\infty}^{2}(x) \text { for a.e. } x \in \Omega_{M} \text {. }
$$

From now on let $M$ be fixed. Note that for every $x \in \Omega_{M}$

$$
u(y)-\left\langle p_{x}, y\right\rangle \leq u(x)-\left\langle p_{x}, x\right\rangle+M|y-x|^{2} \text { for all } y \in \Omega,
$$

or

$$
\tilde{u}(y) \leq \tilde{u}(x)+\left\langle q_{x}, y-x\right\rangle \text { for all } y \in \Omega,
$$

where $\tilde{u}=u-M|\cdot|^{2}$ and $q_{x}=p_{x}-2 M x$. Denoting by $\hat{u}$ the upper concave envelope of $\tilde{u}$ on $\bar{\Omega}$, that is,

$$
\hat{u}(x)=\inf \{p(x): p \text { is affine and } p \geq \tilde{u} \text { on } \Omega\},
$$

we obtain that $\tilde{u}=\hat{u}$ on $\Omega_{M}$, or using the notation in [7], $\Omega_{M} \subset \Gamma$, where $\Gamma=$ $\Gamma_{\tilde{u}}^{+}=\{\tilde{u}=\hat{u}\}$ is the upper contact set of $\tilde{u}$ on $\Omega$. From the Aleksandrov theorem $\hat{u}$ is twice differentiable a.e., that is, there exists $F \subset \Omega$ of full measure such that $\hat{u} \in t_{\infty}^{2}(x)$ for every $x \in F$. Note that $D \tilde{u}=D \hat{u}$ on $\Omega_{M} \cap F$, which yields

$$
|\tilde{u}(y)-\hat{u}(y)| \leq O\left(|y-x|^{2}\right) \text { for every } x \in \Omega_{M} \cap F .
$$

We will show that (1) implies that

$$
|\tilde{u}(y)-\hat{u}(y)| \leq o\left(|y-x|^{2}\right) \text { as } y \rightarrow x \text { for a.e. } x \in \Omega_{M} .
$$

Put $v=\hat{u}-\tilde{u}$ and for $N=1,2, \ldots$ let

$$
\Omega_{M, N}=\left\{x \in \Omega_{M}:|v(y)| \leq N|y-x|^{2} \text { for all } y \in \Omega\right\} .
$$

To prove (2) it is enough to show that for every $N$

$$
|v(y)| \leq o\left(|y-x|^{2}\right) \text { as } y \rightarrow x \text { for a.e. } x \in \Omega_{M, N} .
$$

We will show that this holds for any point of density of $\Omega_{M, N}$. So let $x_{0} \in \Omega_{M, N}$ be a point of density and let $1>\epsilon>0$. Then for all sufficiently small $r$, say $r<\delta$, where $B_{\delta}\left(x_{0}\right) \subset \Omega$,

$$
\frac{\left|B_{r}\left(x_{0}\right) \backslash \Omega_{M, N}\right|}{\left|B_{r}\left(x_{0}\right)\right|}<\epsilon^{n} .
$$


Suppose that $y \in B_{\delta(1-\epsilon)}\left(x_{0}\right)$ and let $r=\left|y-x_{0}\right| /(1-\epsilon)<\delta$. It follows that $B_{\epsilon r}(y) \subset B_{r}\left(x_{0}\right)$ and from (4) $B_{\epsilon r}(y) \cap \Omega_{M, N} \neq \emptyset$, say $x_{1} \in B_{\epsilon r}(y) \cap \Omega_{M, N}$. Then

$$
|v(y)| \leq N\left|y-x_{1}\right|^{2}<N \epsilon^{2} r^{2}=\epsilon \frac{N \epsilon}{(1-\epsilon)^{2}}\left|y-x_{0}\right|^{2},
$$

and (3), and consequently (2), follows.

To finish the proof of the theorem it is enough to remark that if $\hat{u} \in t_{\infty}^{2}(x)$ and $|\tilde{u}(y)-\hat{u}(y)| \leq o\left(|y-x|^{2}\right)$ as $y \rightarrow x$, then the Taylor expansion for $\hat{u}$ works for $\tilde{u}$, and thus $\tilde{u} \in t_{\infty}^{2}(x)$, and consequently $u \in t_{\infty}^{2}(x)$.

Remark 2. Under the assumptions of Theorem 1 we proved that for a.e. $x_{0} \in \Omega$ there exist $p\left(x_{0}\right) \in \mathbb{R}^{n}$ and a symmetric $n \times n$ matrix $A\left(x_{0}\right)$ such that

$$
\begin{aligned}
u(y)=u\left(x_{0}\right)+\left\langle p\left(x_{0}\right), y-x_{0}\right\rangle+\frac{1}{2}\left\langle A\left(x_{0}\right)\left(y-x_{0}\right),\right. & \left.y-x_{0}\right\rangle \\
& +o\left(\left|y-x_{0}\right|^{2}\right) \text { as } y \rightarrow x_{0} .
\end{aligned}
$$

Clearly $u$ is then differentiable at every such point $x_{0}$ with $D u\left(x_{0}\right)=p\left(x_{0}\right)$. A natural question arises whether $A(x)$ is the derivative of $D u(x)$. Denoting $F_{1}=$ $\{x \in \Omega: D u(x)$ exists $\}$ and $F_{2}=\left\{x \in \Omega: u \in t_{\infty}^{2}(x)\right\} \subset F_{1}$, we would like to find out whether for a.e. $x_{0} \in F_{2}$

$$
D u(y)=D u\left(x_{0}\right)+\left\langle A\left(x_{0}\right), y-x_{0}\right\rangle+o\left(\left|y-x_{0}\right|\right) \text { as } F_{1} \ni y \rightarrow x_{0} .
$$

By the $C^{2}$ version of the Aleksandrov theorem (see e.g. [10] or [4]) convex functions have this property, and therefore the proof of Theorem 1 shows that (6) holds in the approximate sense for a.e. $x_{0} \in \Omega$. That is, there exists $F_{3} \subset F_{2} \subset \Omega$ of full measure such that for every $x_{0} \in F_{3}$ and $\epsilon>0$ the set

$$
\left\{y \in F_{1}:\left|D u(y)-D u\left(x_{0}\right)-\left\langle A\left(x_{0}\right), y-x_{0}\right\rangle\right|<\epsilon\left|y-x_{0}\right|\right\}
$$

has density 1 at $x_{0}$. In general, to claim (6) stronger assumptions on $u$ are required; see e.g. Theorem 3.5.7 in [17].

We would like to emphasize that this paper is concerned with pointwise derivatives and in general in our setting one doesn't expect the existence of generalized derivatives. However, if $u \in t_{\infty}^{2}(x)$ for all $x \in \Omega$ with $p$ and $A$ as in (5) belonging to $L^{p}(\Omega), 1 \leq p<\infty$, then $u \in W^{2, p}(\Omega)$; see Theorem 3.9.5 in [17].

A modification of our approach leads to a proof of a parabolic version of the generalized Stepanov theorem. We are concerned with real-valued functions on $\mathbb{R}^{n+1}$. We will write points in $\mathbb{R}^{n+1}$ as $(x, t)$, where $x \in \mathbb{R}^{n}$ and $t \in \mathbb{R}$. Given $(y, s),(x, t) \in \mathbb{R}^{n+1}$, define their parabolic distance $d$ according to

$$
d((y, s),(x, t))=\sqrt{|x-y|^{2}+|t-s|}
$$

and their one-sided parabolic distance $d_{\infty}$ by

$$
d_{\infty}((y, s),(x, t))=\left\{\begin{array}{l}
d((y, s),(x, t)) \text { if } s \leq t \\
+\infty \text { otherwise }
\end{array}\right.
$$

Let $u: Q \rightarrow \mathbb{R}, Q \subset \mathbb{R}^{n+1}$, be bounded and $\left(x_{0}, t_{0}\right) \in Q$. We say that $u \in$ $T_{\infty}^{2,1}\left(x_{0}, t_{0}\right)\left(u \in t_{\infty}^{2,1}\left(x_{0}, t_{0}\right)\right.$, resp. $)$ if there exists an affine function $P_{x_{0}, t_{0}}$ of variable 
$x$ (a quadratic in $x$ and affine in $t$ function $Q_{x_{0}, t_{0}}$ ) such that

$$
\begin{gathered}
\left|u(y, s)-P_{x_{0}, t_{0}}(y, s)\right| \leq O\left(d_{\infty}^{2}\left((y, s),\left(x_{0}, t_{0}\right)\right)\right) \text { for }(y, s) \in Q \\
\left(\left|u(y, s)-Q_{x_{0}, t_{0}}(y, s)\right| \leq o\left(d^{2}\left((y, s),\left(x_{0}, t_{0}\right)\right)\right) \text { as } Q \ni(y, s) \rightarrow\left(x_{0}, t_{0}\right), \text { resp. }\right) .
\end{gathered}
$$

Note that in the definition of $t_{\infty}^{2,1}\left(x_{0}, t_{0}\right)$ an appropriate inequality holds for $s$ both larger and smaller than $t_{0}$, while in the definition of $T_{\infty}^{2,1}\left(x_{0}, t_{0}\right)$ only the values $s \leq t_{0}$ matter. $u \in t_{\infty}^{2,1}\left(x_{0}, t_{0}\right)$ roughly corresponds to the differentiability of $u$ at $\left(x_{0}, t_{0}\right)$, twice in $x$, once in $t$.

Theorem 3. Let $\Omega \subset \mathbb{R}^{n}$ be open and bounded, $T>0$ and suppose that $u: \bar{Q} \rightarrow \mathbb{R}$ is bounded, where $Q=\Omega \times(0, T)$. If $u \in T_{\infty}^{2,1}(x, t)$ for a.e. $(x, t) \in Q$ then $u \in t_{\infty}^{2,1}(x, t)$ for a.e. $(x, t) \in Q$.

$$
\begin{aligned}
& \text { For }(x, t) \in \mathbb{R}^{n+1} \text { and } r>0 \text { put } \\
& \qquad \begin{aligned}
P_{r}(x, t)=\left\{(y, s) \in \mathbb{R}^{n+1}: d((x, t),(y, s))<r\right\}, \\
Q_{r}(x, t)=\left\{(y, s) \in \mathbb{R}^{n+1}: d_{\infty}((x, t),(y, s))<r\right\} .
\end{aligned}
\end{aligned}
$$

The following proposition will be used in the proof of Theorem 3. It follows in a standard way from a version of the covering theorem of Vitali, which employs $Q_{r}$ 's instead of the Euclidean balls; see e.g. Remark I.3.1 in [8].

Proposition 4. Let $A \subset \mathbb{R}^{n+1}$ be measurable. Then

$$
\lim _{r \downarrow 0} \frac{\left|Q_{r}(x, t) \backslash A\right|}{\left|Q_{r}(x, t)\right|}=0 \text { for a.e. }(x, t) \in A .
$$

Proof of Theorem 3. The proof of Theorem 3 parallels that of Theorem 1. By assumption for a.e. $(x, t) \in Q$ there are $p_{x, t} \in \mathbb{R}^{n}$ and $M_{x, t} \geq 0$ such that

$$
\left|u(y, s)-u(x, t)-\left\langle p_{x, t}, y-x\right\rangle\right| \leq M_{x, t}\left(|y-x|^{2}+t-s\right) \text { for all } y \in \Omega, s \in[0, t] .
$$

As before the mapping $(x, t) \mapsto M_{x, t}$ is measurable and putting for $M=1,2, \ldots$

$$
Q_{M}=\left\{(x, t) \in Q: M_{x, t} \leq M\right\}
$$

gives that $\cup_{M=1}^{\infty} Q_{M}$ is of full measure in $Q$. Fix $M$ and define $\tilde{u}(x, t)=u(x, t)-$ $M\left(|x|^{2}-t\right)$; it follows that for every $(x, t) \in Q_{M}$

$$
\tilde{u}(y, s) \leq \tilde{u}(x, t)+\langle q, y-x\rangle \text { for all } y \in \Omega \text { and } s \in[0, t],
$$

with an appropriate $q \in \mathbb{R}^{n}$. In the parabolic context the upper concave envelope $\hat{u}$ of given function $\tilde{u}: Q \rightarrow \mathbb{R}$ is defined by (see [13] or [15])

$$
\hat{u}=\inf \{v: v \geq \tilde{u} \text { on } Q, v \text { concave in } x \text { and increasing in } t\},
$$

and thus (7) shows that $\tilde{u}=\hat{u}$ on $Q_{M}$. A parabolic version of the Aleksandrov theorem (see Theorem 1, Appendix 2 in [9]) guarantees that there exists $F \subset Q$ of full measure such that $\hat{u} \in t_{\infty}^{2,1}(x, t)$ for every $(x, t) \in F$. It follows that

$$
|\tilde{u}(y, s)-\hat{u}(y, s)| \leq O\left(d_{\infty}^{2}((y, s),(x, t))\right) \quad \text { for every }(x, t) \in Q_{M} \cap F .
$$

We will show that (8) implies that

$$
|\tilde{u}(y, s)-\hat{u}(y, s)| \leq o\left(d^{2}((y, s),(x, t))\right) \text { for a.e. }(x, t) \in Q_{M},
$$


which will give the result as in the proof of Theorem 1. Put $v=\hat{u}-\tilde{u}$ and for $N=1,2, \ldots$ let

$$
Q_{M, N}=\left\{(x, t) \in Q_{M}:|v(y, s)| \leq N d_{\infty}^{2}((y, s),(x, t)) \text { for every }(y, s) \in Q\right\},
$$

and suppose that $\left(x_{0}, t_{0}\right) \in Q_{M, N}$ is such that

$$
\lim _{r \downarrow 0} \frac{\left|Q_{r}\left(x_{0}, t_{0}\right) \backslash Q_{M, N}\right|}{\left|Q_{r}\left(x_{0}, t_{0}\right)\right|}=0 ;
$$

by Proposition 4 a.e. $\left(x_{0}, t_{0}\right) \in Q_{M, N}$ will do. Let $0<\epsilon<1$. For all sufficiently small $r$, say $r<\delta$,

$$
\frac{\left|Q_{r}\left(x_{0}, t_{0}\right) \backslash Q_{M, N}\right|}{\left|Q_{r}\left(x_{0}, t_{0}\right)\right|}<\epsilon^{n+2} .
$$

Suppose that $(y, s) \in P_{\delta(1-\epsilon)}\left(x_{0}, t_{0}\right)$ and let $r=d\left((y, s),\left(x_{0}, t_{0}\right)\right) /(1-\epsilon)<\delta$. It follows that $P_{\epsilon r}(y, s) \subset P_{r}\left(x_{0}, t_{0}\right)$ and from (10) $Q_{\epsilon r}(y, s) \cap Q_{M, N} \neq \emptyset$, say $\left(x_{1}, t_{1}\right) \in Q_{\epsilon r}(y, s) \cap Q_{M, N}$. In particular $t_{1} \geq s$ and therefore

$$
|v(y, s)| \leq N\left(\left|y-x_{1}\right|^{2}+t_{1}-s\right)<N \epsilon^{2} r^{2}=\epsilon \frac{N \epsilon}{(1-\epsilon)^{2}} d^{2}\left((y, s),\left(x_{0}, t_{0}\right)\right) .
$$

Thus (9) is proved for a.e. $(x, t) \in Q_{M, N}$ for every $N$, and consequently for a.e. $(x, t) \in Q_{M}$.

\section{REFERENCES}

1. A. D. Aleksandrov, Almost everywhere existence of the second differential of a convex function and some properties of convex functions, Leningrad Univ. Ann. (Math. Ser.) 37 (1939), 3-35 (Russian).

2. L. Caffarelli, M. G. Crandall, M. Kocan, and A. Święch, On viscosity solutions of fully nonlinear equations with measurable ingredients, Comm. Pure Appl. Math. 49 (1996), 365-397. CMP 96:09

3. A. P. Calderón and A. Zygmund, Local properties of solutions of elliptic partial differential equations, Studia Math. 20 (1961), 171-225. MR 25:310

4. M. G. Crandall, H. Ishii, and P.L. Lions, User's guide to viscosity solutions of second order partial differential equations, Bull. Amer. Math. Soc. 27 (1992), 1-67. MR 92j:35050

5. L. C. Evans and R. F. Gariepy, Measure theory and fine properties of functions, CRC Press, Boca Raton, 1992. MR 93f: 28001

6. H. Federer, Geometric measure theory, Springer-Verlag, New York, 1969. MR 41:1976

7. D. Gilbarg and N. S. Trudinger, Elliptic partial differential equations of second order, 2nd ed., Springer-Verlag, New York, 1983. MR 86c:35035

8. M. de Guzmán, Differentiation of integrals in $\mathbb{R}^{n}$, Lecture Notes in Math., vol. 481, SpringerVerlag, New York, 1975. MR 56:15866

9. N. V. Krylov, Nonlinear elliptic and parabolic equations of the second order, Reidel Pub. Co., Dordrecht, 1987. MR 88d:35005

10. F. Mignot, Contrôle optimal dans les inéqualitions variationelles ellitiques, J. Funct. Anal. 22 (1976), 130-185. MR 54:11136

11. N. S. Nadirashvili, Some differentiability properties of solutions of elliptic equations with measurable coefficients, Math. USSR Izvestiya 27 (1986), 601-606.

12. W. H. Oliver, Differential properties of real functions, Ph.D. thesis, Univ. of Chicago, 1951.

13. S. J. Reye, Fully non-linear parabolic differential equations of second order, Ph.D. thesis, Australian National Univ., 1985.

14. N. S. Trudinger, On the twice differentiability of viscosity solutions of nonlinear elliptic equations, Bull. Austral. Math. Soc. 39 (1989), 443-447. MR 90f:35038 
15. Kaising Tso, On an Aleksandrov-Bakel'man type maximum principle for second-order parabolic equations, Comm. Part. Diff. Eq. 10 (1985), 543-553. MR 87f:35031

16. Lihe Wang, On the regularity of fully nonlinear parabolic equations: I, Comm. Pure Appl. Math. 45 (1992), 27-76. MR 92m:35126

17. W. P. Ziemer, Weakly differentiable functions, Springer-Verlag, New York, 1989. MR 91e: 46046

Centre for Mathematics and Its Applications, Australian National University, CanBerra, ACT 0200, Australia

E-mail address: kocan@maths.anu.edu.au

E-mail address: wang@maths.anu.edu.au 